\title{
RADIATION AND THE STRUCTURE OF BONE
}

\author{
F. W. Spiers and G. W. ReED
}

Whenever the body is irradiated, for diagnostic or therapeutic purposes, a considerable amount of bone and bone marrow is inevitably included in the field of the radiation beam. Furthermore, many of the radioisotopes used clinically become incorporated in bone, sometimes to a high degree. The physical consideration of this irradiation of bone is complex because (a) the increased radiation absorption in bone, compared with that in soft tissues, depends markedly on radiation energy, (b) the deposition of radioisotopes in bone is frequently very non-uniform and (c) the ionizing particles (secondary electrons released by X-rays and $\alpha$ and $\beta$ particles from radioisotopes) which distribute the radiation energy within bone, have ranges which are of the same order of magnitude as the dimensions of the microscopic structures of the bone anatomy. Three facets of studies relating to bone will be described, two of which are also of interest outside the immediate field of radiology.

\section{Radiation Dose in Bone}

Although the crystals of hydroxyapatite are distributed discontinuously in a matrix of collagen fibres and ground substance, this mineralized matrix can be regarded for the present purpose as a uniform medium. It has an average or 'effective' atomic number about twice that of soft tissues; it is the component in which the high absorption of radiation takes place and it is the structure in which most of the bone-seeking radioisotopes become lodged. The matrix is not itself very radiosensitive but it acts as the base from which ionizing particles emerge to irradiate other target tissues. These are the soft-tissue structures within bone: (a) the osteocytes, (b) the osteoblasts and other cells lining bone surfaces, such as the periosteum, endosteum, the walls of the Haversian and Volkmann canals and the trabecular surfaces, and (c) the bone marrow itself.

The distribution of the radiation dose within these structures has had to be obtained by calculation because physical measurements of dose over distances of a few tens of microns have not been possible (Spiers, 1949; 1953; Woodard and Spiers 1953). Nevertheless, considerable improvements and extensions of these calculations have been made and results of analytical methods are available to give the distribution of dose near plane surfaces and within cylindrical and spherical spaces inside the bone matrix (Kononenko,
1957; Charlton and Cormack, 1962; Hindmarsh, Owen, Vaughan, Lamerton and Spiers, 1958). Some experimental work has also been done which, in effect, tests the theoretical calculations under simplified geometrical conditions on a macroscopic scale. One experiment has been made at the microscopic level by using $T_{4}$ bacteriophage as a biological indicator of the dose inside glass capillaries (simulated bone cavities) of diameters 2 to 50 microns, although the accuracy thereby attained was insufficient for the purposes of a very stringent test (Aspin and Johns, 1963).

In this laboratory we have used 'artificial' bone surfaces and cylinders, impregnated with very small amounts of strontium-9o, and have measured the dose over distances of up to $3 \mathrm{~mm}$. by means of thin discs of a plastic scintillator (100 to 300 microns thick) with electronic measurement of the scintillations emitted (Spiers and Chesters, I962) This has been possible because the ranges of the ${ }^{90} \mathrm{Sr} \beta$ particles are sufficiently large for a macroscopic experiment. We have thus been able to test the correctness of the mathematical methods and obtain experimental evidence of the radiation dose from this now notorious bone-seeker. We have also made an experimental determination of the dose to 'marrow' in a model of trabecular bone, in which plane 'marrow' cavities of $500 \mu$ thickness were sandwiched between plane 'trabeculæ' $125 \mu$ thick containing ${ }^{90} \mathrm{Sr}$ and obtained results which agree to about $10 \%$ with values calculated for a nearly similar model by Engström, Björnerstadt, Clemedson and Nelson (1957).

\section{Structure of Trabecular Bone}

Because the radiation dose to active marrow depends on the dimensions of the marrow cavities in trabecular bone, we have measured by simple optical means the numbers and sizes of marrow cavities as seen in cut sections of various bones (Jacobs and Spiers, 1962). Distributions of cavity size in trabecular bone have been obtained for the third lumbar vertebra, femur, rib and iliac crest of an adult aged 33 years and for vertebræ of individuals aged from 5 to 95 years. Distributions for a vertebra and iliac crest are shown in Figure 3.

A mean cavity size, weighted for the mass of contained marrow, can be obtained approximately 


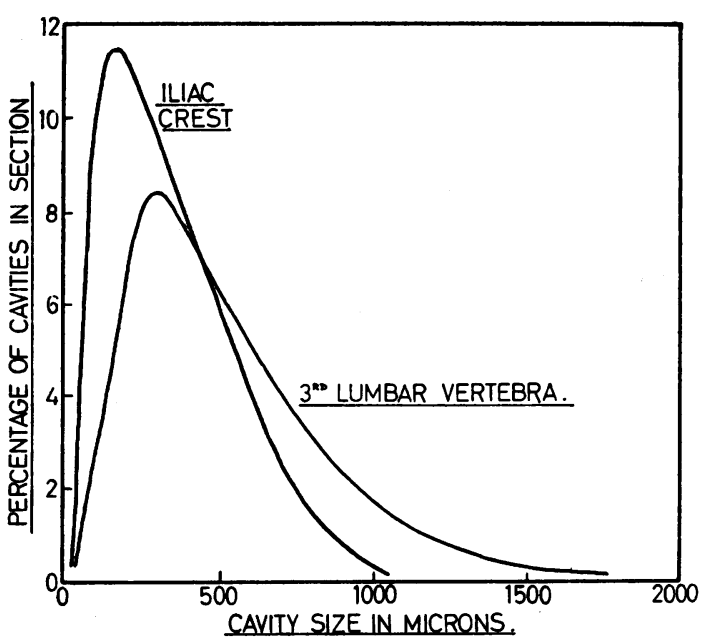

FIG. 3.-Distribution of sizes of marrow cavities in trabecular bone.

by taking the 'mean cube dimension'; these mean dimensions for the lumbar vertebræ are shown in Figure 4 (a) as a function of age. It is possible from these results to calculate approximately the fractions of space in trabecular bone occupied by marrow and hence also the fraction occupied by bone. The bone fraction is shown in Figure 4 (b) from which a picture is obtained of the variation in mineralization of the lumbar spine with age. Clearly, reservations must be made because only a small amount of material has been examined (measuring about 1,000 cavities per section is a time-consuming procedure!) but a maximum mineralization in the young adult is indicated with a decline to possibly half this value in old age. The results obtained in these studies have been used in the determination of the dose to bone marrow in diagnostic X-ray procedures.

\section{Measurement of Bone Mineralization}

Absorption of X-rays of the quality used in diagnostic radiology is strongly dependent on the atomic number of the absorbing atom and various attempts have been made to interpret radiographs of bones quantitatively as a means of measuring calcification. For X-rays of a single energy, the photoelectric absorption per gramme is proportional to the cube of the atomic number and Spiers (1946) developed a laboratory apparatus to select a fixed energy band from an X-ray beam and measure the effective atomic number of cortical bone on the basis of this relationship. Certain radioisotopes now available emit mono-

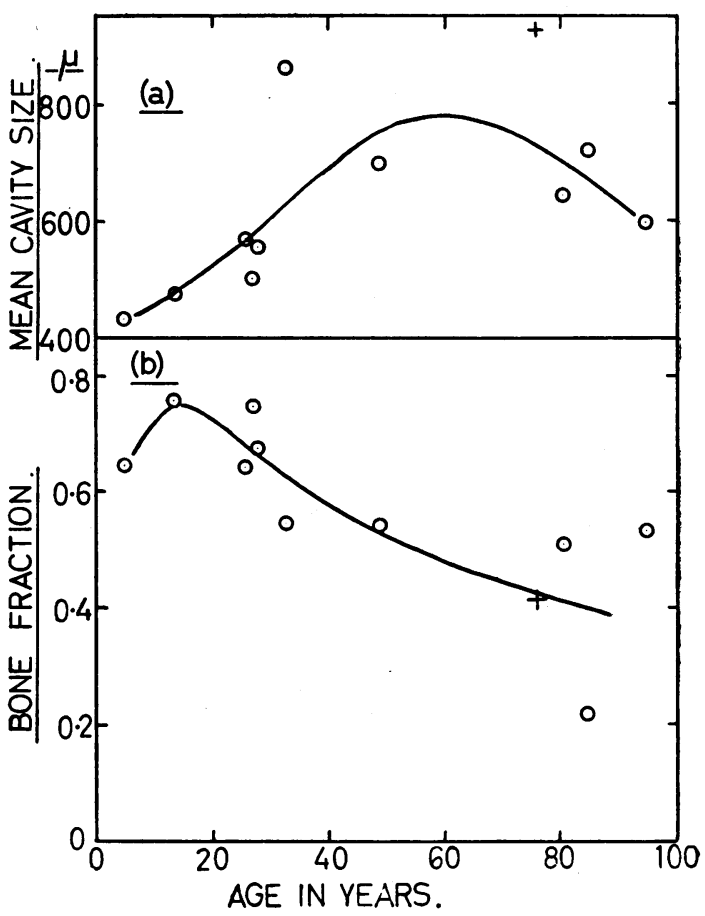

FIG. 4.-(a) Mean sizes of marrow cavities in vertebre of different ages; $(b)$ approximate fraction of space occupied by bone trabeculæ in vertebræ of different5 ages. The cross refers to bone known to be osteoporitic.

energetic radiations which make it possible to make similar measurements on bones in vivo as well as in vitro. Two fine beams of radiation are now used, one from thulium-170 and one from cæsium-137 (Reed, 1963). From the former a peak of energy $84 \mathrm{keV}$ is selected, the absorption of which is dominated by the calcium or other heavy atoms in the beam. The radiation from cæsium-137 is of high energy and its absorption is not sensitive to the atomic number of the absorber, but only to the mass of material present. By measuring with a scintillator detector the absorptions when first one and then the other beam is passed through a bone or joint (e.g. the distal end of the femur) it is possible to determine the amount of calcium in the path traversed. Another isotope, americium-24I, which has very recently become available, can now be used with advantage in place of thulium-170. In vitro tests have proved the feasibility of the apparatus which is now designed for clinical use. Measurements on the sample of cortical bone investigated by Spiers (1946) gave the effective atomic number as 13.7 compared with the value 13.8 determined in the earlier experiments. 


\title{
REFERENCES
}

Aspin, N., and Johns, H. E. (1963): The Absorbed Dose in Cylindrical Cavities within Irradiated Bone, Brit. F.Radiol., 36,350 .

Charlton, D. E., and Cormack, D. V. (1962): Energy Distribution in Finite Cavities, Radiat. Res., 17, 34.

Engström, A., Björnerstedt, R., Clemedson, C. J., and Nelson, A. (1957): In 'Bone and Radiostrontium'. Stockholm: Almqvist and Wiksell.

Hindmarsh, M., Owen, M., Vaughan, J., Lamerton, L. F., and Spiers, F. W. (1958): The Relative Hazards of Strontium-90 and Radium-226, Brit. $\mathcal{F}$. Radiol., 3I, 5 I8.

JACOBS, N., and SPIERS, F. W. (1962): Unpublished data.

Kononenko, A. M. (1957): Calculation of the Dose-rate Produced by Alpha Rays when a Radioactive Substance is Distributed within the Body, Biofyzika (Academy U.S.S.R.), II (I), 94.

REED, G. W. (1963): Unpublished method.

SPIERS, F. W. (1946): Effective Atomic Number and Energy Absorption in Tissues, Brit. F. Radiol., r9, 52.

- (1949): The Influence of Energy Absorption and Electron Range on Dosage in Irradiated Bone, Ibid., $22,521$. (1953): Alpha Ray Dosage in Bone Containing Radium, Ibid., 26, 296.

- and Chesters, M. S. (1962): Theoretical and Experimental Dosimetry Based on Models of Bone Structure. In 'Some Aspects of Internal Irradiation', p. 423. Oxford, London, New York and Paris: Pergamon Press.

Woodard, H. Q., and SPIERS, F. W. (1953): The Effect of X-rays of Different Qualities on the Alkaline Phosphatase of Living Mouse Bone, Brit. F. Radiol., 26, 38.

\section{POSSIBLE AETIOLOGICAL SIGNIFICANCE OF THE AGE-PATTERN OF CERTAIN DISEASES}

\author{
P. R. J. BURCH
}

An interesting feature of disease is its agedistribution. This is commonly described in two ways: ( 1 ) age-specific and sex-specific onset (or mortality) rate-which defines the proportion of people, by sex and by age-group, developing for the first time (or dying from) the disease per year; and (2) age-specific and sex-specific prevalencewhich describes the proportion of people, by sex and by age-group, actually exhibiting the (morbid) disease at a given instant in time.

\section{Malignant Disease}

It has been appreciated for many years that if the logarithm of the age-specific mortality rates for all cancers is plotted against the logarithm of age, a remarkably straight line is obtained for most of adult life. Its slope is about 6 . A similar relationship is found for many particular cancers, and notably for those of epithelial tissues; there are, however, some interesting exceptions to this simple 'rule' including acute leukæmias and bone cancers.

Using this kind of graph, a straight line of slope 6 indicates that age-specific mortality rates are proportional to about the sixth power of age. Unfortunately this attractively simple mathematical relationship can be interpreted in various ways. Nevertheless there is one exceedingly important feature that is common to all the interpretations that have been put forward, and, indeed, to all biologically plausible models: the carcino- genic process involves stochastic phenomena. Ato least two random causal events are needed to explain a sixth power dependence and, on aJ simple view, the maximum number is seven (Muller, 195I; Fisher and Holloman, 195I; Nordling, I953; Stocks, I953; Armitage and Doll, 1954, 1957; Fisher, 1958; Burch, 1962). If the initiation of a cancer depended exclusively on random events, and if the age-specific initiationrate (in the absence of cohort effects) was found to be proportional to the $r^{\text {th }}$ power of age, then $(r+I)$ random events would be required in the initiation process. However, Platt (1955) pointed out that a 'mutant' cell might well enjoy a proliferative advantage over its non-mutant neighbours without actually being malignant. If, therefore, selective growth of mutant cells occurred between one stage of a multi-stage process and the next, the $(r+1)$ rule would no longer apply. For example, if, between the second and the third (and final) random event, 'stage-2 cells' increased in number with the cube of time, then we should find that age-specific initiation rates would be proportional to the fifth power of age although only three random causal events are implicated. All post-Platt models of spontaneous human carcinogenesis have, in fact, incorporated this concept of selective proliferation and it has the by no means negligible advantage of describing, in mathematical terms, the histopathological evidence. for premalignant hyperplasia (Willis, 1960). Any general 